\title{
Synthesis and Cytotoxicity of Silicon and Germanium Containing Pyridine Oxime O-Ethers
}

\author{
Edgars Abele*, Ramona Abele, Pavel Arsenyan, Irina Shestakova, Iveta Kanepe, Inga Antonenko, \\ Juris Popelis, and Edmunds Lukevics* \\ Latvian Institute of Organic Synthesis, \\ 21 Aizkraukles Street, Riga, LV-1006, Latvia; Fax: (371) 7550338; E-mail: abele@osi.lv
}

\begin{abstract}
Silicon and germanium containing pyridine aldoxime, ketoxime and amidoxime O-ethers have been prepared using phase transfer catalytic systems oxime / alkyl halide / solid $\mathrm{KOH}$ / 18-crown- 6 / benzene and oxime / alkyl halide / solid $\mathrm{K}_{2} \mathrm{CO}_{3}$ or $\mathrm{Cs}_{2} \mathrm{CO}_{3}$ / 18-crown-6/toluene. Cytotoxic activity of silicon and germanium containing pyridine oxime O-ethers was tested in vitro on two monolayer tumor cell lines: MG22A (mouse hepatoma) and HT-1080 (human fibrosarcoma). O-[3-Triethylsilylpropyl]- and O-[3-(1-methyl1-silacyclopentyl)propyl] oximes of pyridine aldehydes and ketones exhibit high cytotoxicity. Presence of methyl group in the pyridine ring considerably decreased activity of amidoxime O-ethers. Oxime ethers containing two elements are essentially inactive. For 2-acetylpyridine oxime ethers the activity increases in order of alkyl substituents: $\mathrm{Et}_{3} \mathrm{GeCH}_{2} \mathrm{CH}_{2} \mathrm{SiMe}_{2} \mathrm{CH}_{2}<\mathrm{Et}_{3} \mathrm{SiCH}_{2} \mathrm{CH}_{2} \mathrm{CH}_{2}<\left(\mathrm{CH}_{2}\right)_{4} \mathrm{SiCH}_{2} \mathrm{CH}_{2} \mathrm{CH}_{2}$. Cytotoxicity of ketoxime O-ethers is considerably lower in comparison with aldoxime $\mathrm{O}$-ethers.
\end{abstract}

\section{INTRODUCTION}

Pyridine oxime O-ethers containing different heteroatomic groups are of interest as biologically active compounds. For example, pyridine oxime amino derivatives exhibit antiandrogenic $/ 1,2 /$, antibacterial $/ 3 /$, antidotal against organophosphate poisoning $/ 4,5 /$, fungicidal and pesticidal /6/ activities. Sulfur derivatives of pyridine oxime O-ethers display vasodilating, antiulcer $/ 7 /$, trombocytes aggregation inhibiting $/ 8 /$, antibacterial $/ 9 /$, cytotoxic $/ 9 /$, antidotal of organophosphate poisoning $/ 10 /$, fungicidal $/ 11 /$ and herbicidal $112,13 /$ activities. Phosphorus containing oxime ethers are reactivators of acetylcholinesterase $/ 14 /$ and exhibit insecticidal $/ 15 /$ activity. Fungicidal and insecticidal properties of pyridine aldehyde O-

* Dr. Hab. Chem. Edgars Abele and Prof. Edmunds Lukevics; Latvian Institute of Organic Synthesis, 21 Aizkraukles Street, Riga, LV-1006, Latvia; Tel: (371) 7551822, Fax: (371) 7550338; E-mail: abele@osi.lv 
(triethylstannyl)oximes are also described /16/. Cytotoxicity of silicon and germanium derivatives of pyridine oxime O-ethers has not been investigated till now and is the aim of the present work.

\section{MATERIALS AND METHODS}

\section{Chemistry}

${ }^{1} \mathrm{H}$ NMR spectra were recorded on a Varian 200 Mercury instrument using $\mathrm{CDCl}_{3}$ as solvent and hexamethyldisiloxane (HMDSO) as internal standard. Mass spectra were registered on a GC-MS HP 6890 $(70 \mathrm{eV}) . \mathrm{GC}$ analysis was performed on a Chrom-5 instrument equipped with flame-ionization detector using glass column packed with $5 \%$ OV-101 / Chromosorb W-HP (80-100 mesh) (1.2 m x $3 \mathrm{~mm})$. Pyridine oximes 1-3 and 13-16 were obtained according to the procedures described in /17, 18/. 1-(3-lodopropyl)-1methylsilacyclopentane was obtained by Grignard reaction /19, 20/. $\mathrm{MeEt}_{2} \mathrm{SiCH}_{2} \mathrm{CH}_{2} \mathrm{Si}\left(\mathrm{Me}_{2}\right) \mathrm{CH}_{2} \mathrm{I}$ and $\mathrm{Et}_{3} \mathrm{GeCH}_{2} \mathrm{CH}_{2} \mathrm{Si}\left(\mathrm{Me}_{2}\right) \mathrm{CH}_{2} \mathrm{I}$ were prepared as described in reference $/ 21 /$. The purity of all synthesized compounds was determined by HPLC and content of impurities was not higher than $2.5 \%$.

\section{General procedure for alkylation of pyridine aldoximes and ketoximes 1-3.}

Haloalkylsilane $(1 \mathrm{mmol})$ was added to a suspension of pyridine oxime 1-3 $(1 \mathrm{mmol}), 18$-crown-6 $(0.026$ $\mathrm{g}, 0.1 \mathrm{mmol})$ and powdered $\mathrm{KOH}(0.168 \mathrm{~g}, 3 \mathrm{mmol})$ in benzene $(1 \mathrm{ml})$. The reaction mixture was stirred for 3-6 $\mathrm{h}$ at reflux (GLC control). The resulting mixture was filtered, benzene was removed under reduced pressure and the residue was purified by column chromatography (benzene-ethyl acetate in different ratio was used as eluent) to give products 4-12. The results can be seen in Tables 1, 3 and 4.

\section{General procedure for alkylation of pyridine amidoximes 13-16}

Haloalkylsilane $(1 \mathrm{mmol})$ was added to a suspension of pyridine amidoxime 13-16 $(1 \mathrm{mmol}), 18$-crown-6 $(0.026 \mathrm{~g}, 0.1 \mathrm{mmol})$ and powdered $\mathrm{K}_{2} \mathrm{CO}_{3}\left(0.41 \mathrm{~g}, 3 \mathrm{mmol}\right.$ ) (in the synthesis of compounds $19,20 \mathrm{Cs}_{2} \mathrm{CO}_{3}$ was used) in toluene $(2 \mathrm{ml})$. The reaction mixture was stirred for 6-12 $\mathrm{h}$ at reflux (GLC control). The resulting mixture was filtered, benzene was removed under reduced pressure and the residue was purified by column chromatography (benzene-ethyl acetate in different ratio was used ac eluent) to give products 17-25. The results can be seen in Tables 2-4.

\section{In vitro cytotoxicity assay}

Monolayer cell lines were cultivated for $72 \mathrm{~h}$ in DMEM standard medium without an indicator and antibiotics. After the ampoule was unfrozen not more than four passages were performed. The control cells and cells with tested substances in the range of $(2-5) \times 10^{4}$ cell $/ \mathrm{mL}$ concentration (depending on line nature) were placed on separate 96 wells plates. Solutions containing test compounds were diluted and added in 
wells to give the final concentrations of $50,25,12.5$, and $6.25 \mu \mathrm{g} / \mathrm{mL}$ Control cells were treated in the same manner only in the absence of test compounds. Plates were cultivated for $72 \mathrm{~h}$. The amount of surviving cells was determined using crystal violet (CV) or 3-(4,5-dimethylthiazol-2-yl)-2,5-diphenyltetrazolinium bromide (MTT) coloration, which was assayed by multiscan spectrophotometer. The quantity of live cells on control plate was taken in calculations for $100 \% / 22,23 /$. Concentration of NO was determined according to $/ 22 /$.

\section{RESULTS AND DISCUSSION}

\section{Chemistry}

Alkylation of pyridine oximes with silicon and germanium containing alkyl halides was studied. The known methods for the preparation of oxime O-ethers are based on oxime alkylation in the presence of $\mathrm{NaH} /$ DMF $/ 24$ / or alkali metal alkoxide $/ 25,26 /$. The oxime alkylation reactions in the phase transfer catalytic (PTC) systems oxime / $\mathrm{RX}(\mathrm{X}=\mathrm{Br}, \mathrm{I}) / 10 \%$ aqueous $\mathrm{NaOH} / \mathrm{R} 4 \mathrm{NX}\left(\mathrm{R}=\mathrm{n}-\mathrm{Bu}, \mathrm{n}\right.$-Oct; $\left.\mathrm{X}=\mathrm{Br}, \mathrm{HSO}_{4}\right) /$ $\mathrm{PhH} / 27,28$, oxime / $\mathrm{RX}(\mathrm{X}=\mathrm{Br}, \mathrm{I})$ / solid $\mathrm{K}_{2} \mathrm{CO}_{3} /$ 18-crown-6 / $\mathrm{PhH} / 29 /$, oxime / $\mathrm{RCl} /$ solid $\mathrm{KOH}$ or $\mathrm{K}_{2} \mathrm{CO}_{3}$ / solid $\mathrm{KI} /$ 18-crown- 6 / $\mathrm{PhMe}$ or $\mathrm{PhH} / 30$ / and oxime O-acetate or benzoate / $\mathrm{RX}(\mathrm{X}=\mathrm{Br}, \mathrm{I}) /$ solid $\mathrm{KOH} / 18$-crown- 6 / $\mathrm{PhH} / 31 /$ considerably simplifies the preparation of their O-ethers.

The PTC system iodo- or bromomethylsilane / solid $\mathrm{KOH} /$ 18-crown- 6 / benzene was found to be the most active in the synthesis of oxime ethers $\mathbf{4} \mathbf{- 1 2}$ from pyridine aldoximes and ketoximes $\mathbf{1}$ - $\mathbf{3}$ (Table 1, 3, 4). Synthesis of all pyridine oxime O-ethers proceeded stereoselectively with formation of only $E$-isomers (yields up to $65 \%$ ).

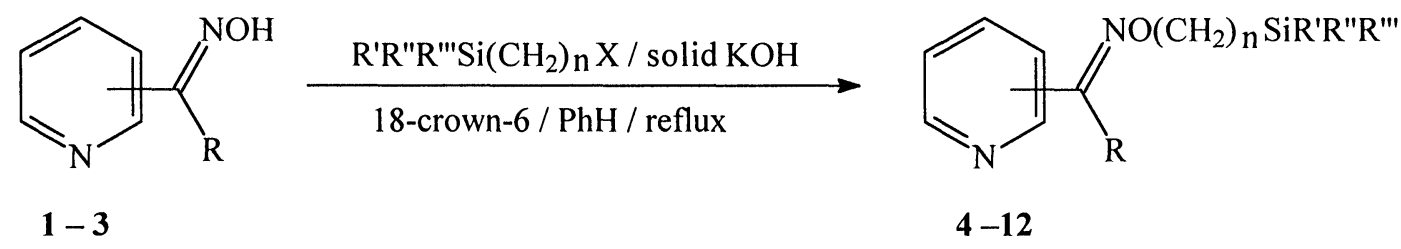

However, the attempts to alkylate the pyridine amidoximes 13-16 with silicon containing alkyl halides in PTC system (solid $\mathrm{KOH} /$ 18-crown-6 / benzene) were uneffective. Replacement of the solid $\mathrm{KOH} / 18$ crown- 6 / benzene to solid $\mathrm{K}_{2} \mathrm{CO}_{3}$ (or $\mathrm{Cs}_{2} \mathrm{CO}_{3}$ ) / 18-crown- 6 / toluene increased the yield of pyridine amidoxime O-ethers 17-25 (Tables 2-4). The $E$-isomers of products 17-25 were isolated by column chromatography in $40-79 \%$ yields.

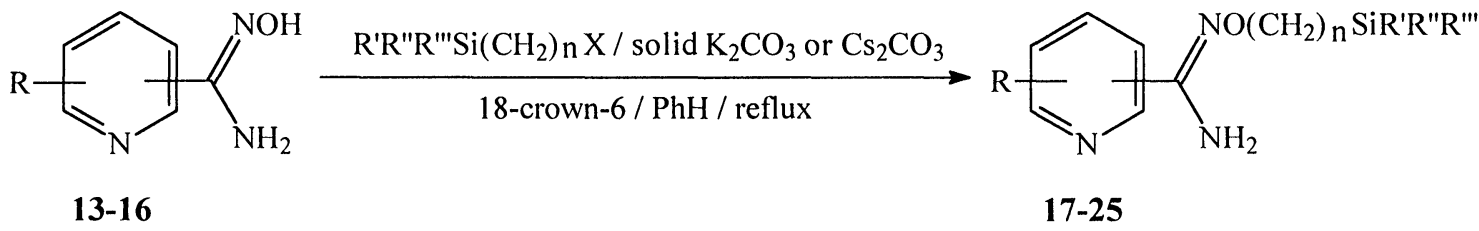


Table 1

Synthesis of pyridine aldoxime and ketoxime O-ethers 4-12

\begin{tabular}{|c|c|c|c|c|c|c|c|c|}
\hline Oxime & Pyridyl & $\mathrm{R}$ & SiR'R"R"' & $\mathrm{n}$ & $X$ & $\begin{array}{c}\text { Reaction } \\
\text { time, } \mathrm{h}\end{array}$ & Product & $\begin{array}{c}\text { Yield }^{\mathrm{a}}, \\
\%\end{array}$ \\
\hline 1 & $2-$ & $\mathrm{H}$ & $\mathrm{Si}\left(\mathrm{Me}_{2}\right) \mathrm{CH}_{2} \mathrm{CH}_{2} \mathrm{GeEt}_{3}$ & 1 & I & 3 & 4 & 42 \\
\hline 1 & $2-$ & $\mathrm{H}$ & $\mathrm{SiEt}_{3}$ & 3 & $\mathrm{Br}$ & 6 & 5 & 48 \\
\hline 1 & $2-$ & $\mathrm{H}$ & 1-methyl-1-silacyclopentyl & 3 & I & 6 & 6 & 31 \\
\hline 2 & $2-$ & $\mathrm{Me}$ & $\mathrm{Si}\left(\mathrm{Me}_{2}\right) \mathrm{CH}_{2} \mathrm{CH}_{2} \mathrm{SiEt}_{2} \mathrm{Me}$ & 1 & I & 3 & 7 & 51 \\
\hline 2 & $2-$ & $\mathrm{Me}$ & $\mathrm{Si}\left(\mathrm{Me}_{2}\right) \mathrm{CH}_{2} \mathrm{CH}_{2} \mathrm{GeEt}_{3}$ & 1 & I & 3 & 8 & 56 \\
\hline 2 & 2- & $\mathrm{Me}$ & $\mathrm{SiEt}_{3}$ & 3 & $\mathrm{Br}$ & 6 & 9 & 41 \\
\hline 2 & $2-$ & $\mathrm{Me}$ & 1-methyl-1-silacyclopentyl & 3 & I & 6 & 10 & 65 \\
\hline 3 & 4- & $\mathrm{H}$ & $\mathrm{SiEt}_{3}$ & 3 & $\mathrm{Br}$ & 6 & 11 & 62 \\
\hline 3 & 4- & $\mathrm{H}$ & 1-methyl-1-silacyclopentyl & 3 & $\mathrm{I}$ & 6 & 12 & 64 \\
\hline
\end{tabular}

${ }^{\text {a }}$ Yields of isolated products

Table 2

Synthesis of pyridine amidoxime O-ethers 17-25

\begin{tabular}{|c|c|c|c|c|c|c|c|c|}
\hline Oxime & Pyridyl & $\mathrm{R}$ & SiR'R"R"' & $\mathrm{n}$ & $X$ & $\begin{array}{c}\text { Time, } \\
\mathrm{h} \\
\end{array}$ & Product & $\begin{array}{c}\text { Yield }^{\mathrm{a}}, \\
\% \\
\end{array}$ \\
\hline 13 & 3- & $\mathrm{H}$ & $\mathrm{SiEt}_{3}$ & 3 & $\mathrm{Br}$ & 6 & 17 & 40 \\
\hline 13 & 3- & $\mathrm{H}$ & 1-methyl-1-silacyclopentyl & 3 & I & 7 & 18 & 6.5 \\
\hline 14 & 4- & $\mathrm{H}$ & $\mathrm{SiEt}_{3}$ & 3 & $\mathrm{Br}$ & 12 & 19 & 40 \\
\hline 14 & 4- & $\mathrm{H}$ & 1-methyl-1-silacyclopentyl & 3 & I & 12 & 20 & 33 \\
\hline 15 & 2 & 3-Me & $\mathrm{SiEt}_{3}$ & 3 & $\mathrm{Br}$ & 8 & 21 & 49 \\
\hline 15 & 2 & 3-Me & 1-methyl-1-silacyclopentyl & 3 & I & 6 & 22 & 59 \\
\hline 15 & 2 & 3-Me & $\mathrm{Si}\left(\mathrm{Me}_{2}\right) \mathrm{CH}_{2} \mathrm{CH}_{2} \mathrm{SiEt}_{2} \mathrm{Me}$ & 1 & I & 8 & 23 & 46 \\
\hline 16 & 2 & 6-Me & $\mathrm{SiEt}_{3}$ & 3 & $\mathrm{Br}$ & 7 & 24 & 60 \\
\hline 16 & 2 & 6-Me & $\mathrm{Si}\left(\mathrm{Me}_{2}\right) \mathrm{CH}_{2} \mathrm{CH}_{2} \mathrm{GeEt}_{3}$ & 1 & $\mathrm{I}$ & 9 & 25 & 64 \\
\hline
\end{tabular}

${ }^{\text {a }}$ Yields of isolated products

\section{In Vitro cytotoxicity}

Cytotoxic activity of silicon and germanium containing pyridine oxime O-ethers was tested in vitro on two monolayer tumor cell lines: MG-22A (mouse hepatoma) and HT-1080 (human fibrosarcoma). Concentrations providing $50 \%$ of tumor death effect were determined according to the known procedure $/ 32 /$ using 96 well plates. 
Table 3

Mass spectroscopic data of pyridine oxime O-ethers

\begin{tabular}{|c|c|}
\hline Compound & $\mathrm{M} / \mathrm{z}$ (intensity, \%) \\
\hline 4 & $\begin{array}{l}337\left(<1, \mathrm{M}^{+}-\mathrm{C}_{3} \mathrm{H}_{7}\right), 219(4), 193(63), 163(100), 149(5), 133(16), 119(9), 103(14), 92 \\
(12), 78(10), 59(21) .\end{array}$ \\
\hline 5 & $\begin{array}{l}279\left(<1, M^{+}\right), 249(23), 233(8), 145(7), 115(21), 103(100), 87(41), 78(74), 59(30), \\
51(14)\end{array}$ \\
\hline 6 & $\begin{array}{l}\left.262\left(2, M^{+}\right), 217(9), 203(89), 192(8), 184(18), 175(17), 16510\right), 148(40), 133(13), \\
119(18), 101(84), 87(19), 78(100), 61(23), 45(37)\end{array}$ \\
\hline 7 & $\begin{array}{l}321\left(<1, \mathrm{M}^{+}-\mathrm{Me}\right), 291(3), 207(51), 177(100), 136(16), 119(13), 101(14), 87(15), 78 \\
(45), 59(26), 45(26\end{array}$ \\
\hline 8 & $\begin{array}{l}367\left(<1, M^{+}-E t\right), 207(73), 177(100), 161(5), 147 \text { (5), } 136(17), 103 \text { (130), } 78 \text { (47), } 59 \\
(16), 43(7)\end{array}$ \\
\hline 9 & $291\left(<1, \mathrm{M}^{+}\right), 247(7), 133(25), 119(72), 87(22), 78(100), 59(17), 51(10)$ \\
\hline 10 & $276\left(<1, \mathrm{M}^{+}\right), 231(5), 133(18), 119(50), 99(18), 78(100), 45(10)$ \\
\hline 11 & $249\left(35, \mathrm{M}^{+}-\mathrm{Et}\right), 115(53), 103(100), 87(62), 75(66), 59(34)$ \\
\hline 12 & $\begin{array}{l}262\left(5, M^{+}\right), 233(5), 184(41), 133(5), 101(100), 87(13), 78(23), 61(13), 51(18), 43 \\
(11)\end{array}$ \\
\hline 17 & $\begin{array}{l}293\left(2, \mathrm{M}^{+}\right), 264(12), 222(4), 137(23), 120(100), 103(68), 87(34), 78(30), 59(28), \\
51(16)\end{array}$ \\
\hline 18 & $\begin{array}{l}276\left(4, M^{+}\right), 248(12), 234(14), 220(13), 193(8), 163(10), 137(20), 129(9), 120(100), \\
102(66), 87(27), 78(66), 59(24), 45(46)\end{array}$ \\
\hline 19 & $\begin{array}{l}293\left(2, M^{+}\right), 264(51), 222(5), 137(23), 120(100), 103(81), 87(43), 78(36), 59(33), \\
43(10)\end{array}$ \\
\hline 20 & $\begin{array}{l}277\left(6, M^{+}\right), 248(47), 234(34), 220(10), 193(8), 178(12), 148(45), 137(33), 129(10), \\
120(93), 102(100), 87(29), 78(77), 59(26), 51(50), 43(45)\end{array}$ \\
\hline 21 & $\begin{array}{l}307\left(2, M^{+}\right), 262(6), 162(13), 151(10), 134(100), 118(16), 103(18), 92(36), 75(13), \\
59(18)\end{array}$ \\
\hline 22 & $\begin{array}{l}290\left(2, M^{+}\right), 276(6), 177(16), 162(22), 145(10), 134(100), 119(26), 101(20), 92(66), \\
65(29), 45(22)\end{array}$ \\
\hline 23 & $\begin{array}{l}306\left(2, \mathrm{M}^{+} \text {-Et-Me), } 222(75), 192(89), 134(13), 119(16), 101(16), 92(20), 74(100), 59\right. \\
(26), 45(25)\end{array}$ \\
\hline 24 & $\begin{array}{l}307\left(3, M^{+}\right), 278(7), 262(10), 178(10), 162(41), 151(24), 134(100), 119(32), 103 \\
(34), 92(66), 75(22), 59(35), 43(12)\end{array}$ \\
\hline 25 & $\begin{array}{l}396\left(<1, M^{+}-\mathrm{Me}\right), 222(100), 192(46), 133(14), 119(28), 103(14), 92(14), 74(97), 59 \\
(17)\end{array}$ \\
\hline
\end{tabular}


Table 4

${ }^{1} \mathrm{H}$ NMR data of pyridine oxime O-ethers

\begin{tabular}{|c|c|}
\hline Ether & $\left.\delta \mathrm{ppm}, \mathrm{CDCl}_{3} / \mathrm{HMDSO}\right)$ \\
\hline 4 & $\begin{array}{l}0.06\left(\mathrm{~s}, 6 \mathrm{H}, \mathrm{Si}\left(\mathrm{CH}_{3}\right)_{2}\right), 0.67\left(\mathrm{~m}, 10 \mathrm{H}, \mathrm{SiCH}_{2} \text { and } \mathrm{GeCH}_{2}\right), 0.99(\mathrm{t}, 9 \mathrm{H}, \mathrm{J}=7,6 \mathrm{~Hz}, \\
\left.\mathrm{GeCH}_{2} \mathrm{CH}_{3}\right), 4.06\left(\mathrm{~s}, 2 \mathrm{H}, \mathrm{OCH}_{2}\right), 7.23(\mathrm{~m}, 1 \mathrm{H}, 5-\mathrm{H}), 7.62-7.79(\mathrm{~m}, 2 \mathrm{H}, 3-\mathrm{H} \text { and } 4-\mathrm{H}), \\
8.13(\mathrm{~s}, 1 \mathrm{H}, \mathrm{CH}), 8.59(\mathrm{~m}, 1 \mathrm{H}, 6-\mathrm{H})\end{array}$ \\
\hline 5 & $\begin{array}{l}0.52\left(\mathrm{~m}, 8 \mathrm{H}, \mathrm{SiCH}_{2}\right), 0.92\left(\mathrm{t}, 9 \mathrm{H}, \mathrm{J}=7.4 \mathrm{~Hz}, \mathrm{CH}_{3}\right), 1.60\left(\mathrm{~m}, 2 \mathrm{H}, \mathrm{CH}_{2} \mathrm{CH}_{2} \mathrm{CH}_{2}\right), 4.16(\mathrm{t}, \\
\left.2 \mathrm{H}, \mathrm{J}=7.0 \mathrm{~Hz}, \mathrm{OCH}_{2}\right), 7.26(\mathrm{~m}, 1 \mathrm{H}, 5-\mathrm{H}), 7.62-7.80(\mathrm{~m}, 2 \mathrm{H}, 3-\mathrm{H} \text { and } 4-\mathrm{H}), 8.16(\mathrm{~s}, 1 \mathrm{H}, \\
\mathrm{CH}), 8.61(\mathrm{~m}, 1 \mathrm{H}, 6-\mathrm{H})\end{array}$ \\
\hline 6 & $\begin{array}{l}0.08\left(\mathrm{~s}, 3 \mathrm{H}, \mathrm{CH}_{3}\right), 0.59\left(\mathrm{~m}, 6 \mathrm{H}, \mathrm{SiCH}_{2}\right), 1.55\left(\mathrm{~m}, 6 \mathrm{H}, \mathrm{CH}_{2}\left(\mathrm{CH}_{2}\right)_{2} \mathrm{CH}_{2} \text { in silacycle and }\right. \\
\left.\mathrm{CH}_{2} \mathrm{CH}_{2} \mathrm{CH}_{2}\right), 4.18\left(\mathrm{t}, 2 \mathrm{H}, \mathrm{J}=7.0 \mathrm{~Hz}, \mathrm{OCH}_{2}\right), 7.25(\mathrm{~m}, 1 \mathrm{H}, 5-\mathrm{H}), 7.67-7.80(\mathrm{~m}, 2 \mathrm{H}, 3-\mathrm{H} \\
\text { and } 4-\mathrm{H}), 8.16(\mathrm{~s}, 1 \mathrm{H}, \mathrm{CH}), 8.60(\mathrm{~m}, 1 \mathrm{H}, 6-\mathrm{H})\end{array}$ \\
\hline 7 & $\begin{array}{l}-0.09\left(\mathrm{~s}, 3 \mathrm{H}, \mathrm{SiCH}_{3}\right), 0.10\left(\mathrm{~s}, 6 \mathrm{H}, \mathrm{Si}\left(\mathrm{CH}_{3}\right)_{2}\right), 0.51\left(\mathrm{~m}, 8 \mathrm{H}, \mathrm{SiCH}_{2}\right), 0.91(\mathrm{t}, 6 \mathrm{H}, \mathrm{J}=7,6 \mathrm{~Hz}, \\
\left.\mathrm{SiCH}_{2} \mathrm{CH}_{3}\right), 2.31\left(\mathrm{~s}, 3 \mathrm{H}, \mathrm{CCH}_{3}\right), 4.06\left(\mathrm{~s}, 2 \mathrm{H}, \mathrm{OCH}_{2}\right), 7.36(\mathrm{~m}, 1 \mathrm{H}, 5-\mathrm{H}), 7.45(\mathrm{~m}, 1 \mathrm{H}, 3- \\
\mathrm{H}), 7.90(\mathrm{~m}, 1 \mathrm{H}, 4-\mathrm{H}), 8.59(\mathrm{~m}, 1 \mathrm{H}, 6-\mathrm{H})\end{array}$ \\
\hline 8 & $\begin{array}{l}0.10\left(\mathrm{~s}, 6 \mathrm{H}, \mathrm{Si}\left(\mathrm{CH}_{3}\right)_{2}\right), 0.72\left(\mathrm{~m}, 10 \mathrm{H}, \mathrm{SiCH}_{2} \text { and } \mathrm{GeCH}_{2}\right), 0.99(\mathrm{t}, 9 \mathrm{H}, \mathrm{J}=7.2 \mathrm{~Hz}, \\
\left.\mathrm{GeCH}_{2} \mathrm{CH}_{3}\right), 2.29\left(\mathrm{~s}, 3 \mathrm{H}, \mathrm{CCH}_{3}\right), 4.05\left(\mathrm{~s}, 2 \mathrm{H}, \mathrm{OCH}_{2}\right), 7.21(\mathrm{~m}, 1 \mathrm{H}, 5-\mathrm{H}), 7.64(\mathrm{~m}, 1 \mathrm{H}, 3- \\
\mathrm{H}), 7.89(\mathrm{~m}, 1 \mathrm{H}, 4-\mathrm{H}), 8.58(\mathrm{~m}, 1 \mathrm{H}, 6-\mathrm{H})\end{array}$ \\
\hline 9 & $\begin{array}{l}0.54\left(\mathrm{~m}, 8 \mathrm{H}, \mathrm{SiCH}_{2}\right), 0.93\left(\mathrm{t}, 9 \mathrm{H}, \mathrm{J}=7.6 \mathrm{~Hz}, \mathrm{CH}_{3}\right), 1.60\left(\mathrm{~m}, 2 \mathrm{H}, \mathrm{CH}_{2} \mathrm{CH}_{2} \mathrm{CH}_{2}\right), 2.35(\mathrm{~s}, \\
\left.3 \mathrm{H}, \mathrm{CH}_{3}\right), 4.17\left(\mathrm{t}, 2 \mathrm{H}, \mathrm{J}=7.0 \mathrm{~Hz}, \mathrm{OCH}_{2}\right), 7.25(\mathrm{~m}, 1 \mathrm{H}, 5-\mathrm{H}), 7.61(\mathrm{~m}, 1 \mathrm{H}, 3-\mathrm{H}), 7.87(\mathrm{~m}, \\
1 \mathrm{H}, 4-\mathrm{H}), 8.58(\mathrm{~m}, 1 \mathrm{H}, 6-\mathrm{H})\end{array}$ \\
\hline 10 & $\begin{array}{l}0.09\left(\mathrm{~s}, 3 \mathrm{H}, \mathrm{CH}_{3}\right), 0.60\left(\mathrm{~m}, 6 \mathrm{H}, \mathrm{SiCH}_{2}\right), 1.55\left(\mathrm{~m}, 6 \mathrm{H}, \mathrm{CH}_{2}\left(\mathrm{CH}_{2}\right)_{2} \mathrm{CH}_{2} \text { in silacycle and }\right. \\
\left.\mathrm{CH}_{2} \mathrm{CH}_{2} \mathrm{CH}_{2}\right), 2.32\left(\mathrm{~s}, 3 \mathrm{H}, \mathrm{CH}_{3}\right), 4.18\left(\mathrm{t}, 2 \mathrm{H}, \mathrm{J}=7.0 \mathrm{~Hz}, \mathrm{OCH}_{2}\right), 7.25(\mathrm{~m}, 1 \mathrm{H}, 5-\mathrm{H}), 7.65 \\
(\mathrm{~m}, 1 \mathrm{H}, 3-\mathrm{H}), 7.89(\mathrm{~m}, 1 \mathrm{H}, 4-\mathrm{H}), 8.59(\mathrm{~m}, 1 \mathrm{H}, 6-\mathrm{H})\end{array}$ \\
\hline 11 & $\begin{array}{l}0.54\left(\mathrm{~m}, 8 \mathrm{H}, \mathrm{SiCH}_{2}\right), 0.93\left(\mathrm{t}, 9 \mathrm{H}, \mathrm{J}=7.6 \mathrm{~Hz}, \mathrm{CH}_{3}\right), 1.65\left(\mathrm{~m}, 2 \mathrm{H}, \mathrm{CH}_{2} \mathrm{CH}_{2} \mathrm{CH}_{2}\right), 4.15(\mathrm{t}, \\
\left.2 \mathrm{H}, \mathrm{J}=7.0 \mathrm{~Hz}, \mathrm{OCH}_{2}\right), 7.42(\mathrm{~m}, 2 \mathrm{H}, 3-\mathrm{H} \text { and } 5-\mathrm{H}), 8.01(\mathrm{~s}, 1 \mathrm{H}, \mathrm{CH}), 8.62(\mathrm{~m}, 2 \mathrm{H}, 2-\mathrm{H} \\
\text { and } 6-\mathrm{H})\end{array}$ \\
\hline 12 & $\begin{array}{l}0.09\left(\mathrm{~s}, 3 \mathrm{H}, \mathrm{CH}_{3}\right), 0.59\left(\mathrm{~m}, 6 \mathrm{H}, \mathrm{SiCH}_{2}\right), 1.55\left(\mathrm{~m}, 6 \mathrm{H}, \mathrm{CH}_{2}\left(\mathrm{CH}_{2}\right)_{2} \mathrm{CH}_{2} \text { in silacycle and }\right. \\
\left.\mathrm{CH}_{2} \mathrm{CH}_{2} \mathrm{CH}_{2}\right), 4.16\left(\mathrm{t}, 2 \mathrm{H}, \mathrm{J}=7.0 \mathrm{~Hz}, \mathrm{OCH}_{2}\right), 7.43(\mathrm{~m}, 2 \mathrm{H}, 3-\mathrm{H} \text { and } 5-\mathrm{H}), 8.01(\mathrm{~s}, 1 \mathrm{H}, \\
\mathrm{CH}), 8.62(\mathrm{~m}, 2 \mathrm{H}, 2-\mathrm{H} \text { and } 6-\mathrm{H})\end{array}$ \\
\hline 17 & $\begin{array}{l}0.52\left(\mathrm{~m}, 8 \mathrm{H}, \mathrm{SiCH}_{2}\right), 0.92\left(\mathrm{t}, 9 \mathrm{H}, \mathrm{J}=7.0 \mathrm{~Hz}, \mathrm{CH}_{3}\right), 1.66\left(\mathrm{~m}, 2 \mathrm{H}, \mathrm{CH}_{2} \mathrm{CH}_{2} \mathrm{CH}_{2}\right), 4.07(\mathrm{t}, \\
\left.2 \mathrm{H}, \mathrm{J}=7.0 \mathrm{~Hz}, \mathrm{OCH}_{2}\right), 4.77\left(\mathrm{bs}, 2 \mathrm{H}, \mathrm{NH}_{2}\right), 7.22(\mathrm{~m}, 1 \mathrm{H}, 5-\mathrm{H}), 7.91(\mathrm{~m}, 1 \mathrm{H}, 4-\mathrm{H}), 8.53(\mathrm{~m}, \\
1 \mathrm{H}, 6-\mathrm{H}), 8.85(\mathrm{~m}, 1 \mathrm{H}, 2-\mathrm{H})\end{array}$ \\
\hline 18 & $\begin{array}{l}0.26\left(\mathrm{~s}, 3 \mathrm{H}, \mathrm{CH}_{3}\right), 0.76\left(\mathrm{~m}, 6 \mathrm{H}, \mathrm{SiCH}_{2}\right), 1.78\left(\mathrm{~m}, 6 \mathrm{H}, \mathrm{CH}_{2}\left(\mathrm{CH}_{2}\right)_{2} \mathrm{CH}_{2} \text { in silacycle and }\right. \\
\left.\mathrm{CH}_{2} \mathrm{CH}_{2} \mathrm{CH}_{2}\right), 4.21\left(\mathrm{t}, 2 \mathrm{H}, \mathrm{J}=7.2 \mathrm{~Hz}, \mathrm{OCH}_{2}\right), 5.00\left(\mathrm{bs}, 2 \mathrm{H}, \mathrm{NH}_{2}\right), 7.48(\mathrm{~m}, 1 \mathrm{H}, 5-\mathrm{H}), 8.08 \\
(\mathrm{~m}, 1 \mathrm{H}, 4-\mathrm{H}), 8.77(\mathrm{~m}, 1 \mathrm{H}, 6-\mathrm{H}), 8.98(\mathrm{~m}, 1 \mathrm{H}, 2-\mathrm{H})\end{array}$ \\
\hline
\end{tabular}


Table 4 (continued)

\begin{tabular}{|c|c|}
\hline 19 & $\begin{array}{l}0.52\left(\mathrm{~m}, 8 \mathrm{H}, \mathrm{SiCH}_{2}\right), 0.93\left(\mathrm{t}, 9 \mathrm{H}, \mathrm{J}=7.4 \mathrm{~Hz}, \mathrm{CH}_{3}\right), 1.65\left(\mathrm{~m}, 2 \mathrm{H}, \mathrm{CH}_{2} \mathrm{CH}_{2} \mathrm{CH}_{2}\right), 4.06(\mathrm{t} \text {, } \\
\left.2 \mathrm{H}, \mathrm{J}=7.0 \mathrm{~Hz}, \mathrm{OCH}_{2}\right), 4.79\left(\mathrm{bs}, 2 \mathrm{H}, \mathrm{NH}_{2}\right), 7.51(\mathrm{~m}, 2 \mathrm{H}, 3-\mathrm{H} \text { and 5-H), } 8.62(\mathrm{~m}, 2 \mathrm{H}, 2-\mathrm{H} \\
\text { and 6-H) }\end{array}$ \\
\hline 20 & $\begin{array}{l}0.09\left(\mathrm{~s}, 3 \mathrm{H}, \mathrm{CH}_{3}\right), 0.59\left(\mathrm{~m}, 6 \mathrm{H}, \mathrm{SiCH}_{2}\right), 1.55\left(\mathrm{~m}, 6 \mathrm{H}, \mathrm{CH}_{2}\left(\mathrm{CH}_{2}\right)_{2} \mathrm{CH}_{2} \text { in silacycle and }\right. \\
\left.\mathrm{CH}_{2} \mathrm{CH}_{2} \mathrm{CH}_{2}\right), 4.07\left(\mathrm{t}, 2 \mathrm{H}, \mathrm{J}=6.8 \mathrm{~Hz}, \mathrm{OCH}_{2}\right), 4.79\left(\mathrm{bs}, 2 \mathrm{H}, \mathrm{NH}_{2}\right), 7.51(\mathrm{~m}, 2 \mathrm{H}, 3-\mathrm{H} \text { and 5- } \\
\mathrm{H}), 8.63(\mathrm{~m}, 2 \mathrm{H}, 2-\mathrm{H} \text { and 6-H) }\end{array}$ \\
\hline 21 & $\begin{array}{l}0.52\left(\mathrm{~m}, 8 \mathrm{H}, \mathrm{SiCH}_{2}\right), 0.93\left(\mathrm{t}, 9 \mathrm{H}, \mathrm{J}=6.8 \mathrm{~Hz}, \mathrm{CH}_{3}\right), 1.72\left(\mathrm{~m}, 2 \mathrm{H}, \mathrm{CH}_{2} \mathrm{CH}_{2} \mathrm{CH}_{2}\right), 2.56(\mathrm{~s}, \\
3 \mathrm{H}, \mathrm{CH}_{3} \text { in ring), } 4.03\left(\mathrm{t}, 2 \mathrm{H}, \mathrm{J}=7.0 \mathrm{~Hz}, \mathrm{OCH}_{2}\right), 5.47\left(\mathrm{bs}, 2 \mathrm{H}, \mathrm{NH}_{2}\right), 7.09(\mathrm{~m}, 1 \mathrm{H}, 5-\mathrm{H}), \\
7.45(\mathrm{~m}, 1 \mathrm{H}, 4-\mathrm{H}), 8.36(\mathrm{~m}, 1 \mathrm{H}, 6-\mathrm{H})\end{array}$ \\
\hline 22 & $\begin{array}{l}0.08\left(\mathrm{~s}, 3 \mathrm{H}, \mathrm{CH}_{3}\right), 0.60\left(\mathrm{~m}, 6 \mathrm{H}, \mathrm{SiCH}_{2}\right), 1.55\left(\mathrm{~m}, 6 \mathrm{H}, \mathrm{CH}_{2}\left(\mathrm{CH}_{2}\right)_{2} \mathrm{CH}_{2} \text { in silacycle and }\right. \\
\left.\mathrm{CH}_{2} \mathrm{CH}_{2} \mathrm{CH}_{2}\right), 2.59\left(\mathrm{~s}, 3 \mathrm{H}, \mathrm{CH}_{3} \text { in ring), } 4.07\left(\mathrm{t}, 2 \mathrm{H}, \mathrm{J}=6.8 \mathrm{~Hz}, \mathrm{OCH}_{2}\right), 5.50 \text { (bs, } 2 \mathrm{H} \text {, }\right. \\
\left.\mathrm{NH}_{2}\right), 7.17(\mathrm{~m}, 1 \mathrm{H}, 5-\mathrm{H}), 7.53(\mathrm{~m}, 1 \mathrm{H}, 4-\mathrm{H}), 8.40(\mathrm{~m}, 1 \mathrm{H}, 6-\mathrm{H})\end{array}$ \\
\hline 23 & $\begin{array}{l}-0.15\left(\mathrm{~s}, 3 \mathrm{H}, \mathrm{Et}_{2} \mathrm{SiCH}_{3}\right), 0.04\left(\mathrm{~s}, 6 \mathrm{H}, \mathrm{Si}\left(\mathrm{CH}_{3}\right)_{2}\right), 0.43\left(\mathrm{~m}, 8 \mathrm{H}, \mathrm{SiCH}_{2}\right), 0.82(\mathrm{t}, 6 \mathrm{H}, \mathrm{J}=7.6 \\
\left.\mathrm{Hz}, \mathrm{SiCH}_{2} \mathrm{CH}_{3}\right), 2.54\left(\mathrm{~s}, 3 \mathrm{H}, \mathrm{CH}_{3} \text { in ring }\right), 3.90\left(\mathrm{~s}, 2 \mathrm{H}, \mathrm{OCH}_{2}\right), 5.42\left(\mathrm{bs}, 2 \mathrm{H}, \mathrm{NH}_{2}\right), 7.10 \\
(\mathrm{~m}, 1 \mathrm{H}, 5-\mathrm{H}), 7.45(\mathrm{~m}, 1 \mathrm{H}, 4-\mathrm{H}), 8.34(\mathrm{~m}, 1 \mathrm{H}, 6-\mathrm{H})\end{array}$ \\
\hline 24 & $\begin{array}{l}0.53\left(\mathrm{~m}, 8 \mathrm{H}, \mathrm{SiCH}_{2}\right), 0.90\left(\mathrm{t}, 9 \mathrm{H}, \mathrm{J}=6.8 \mathrm{~Hz}, \mathrm{CH}_{3}\right), 1.71\left(\mathrm{~m}, 2 \mathrm{H}, \mathrm{CH}_{2} \mathrm{CH}_{2} \mathrm{CH}_{2}\right), 2.51(\mathrm{~s}, \\
3 \mathrm{H}, \mathrm{CH}_{3} \text { in ring), } 4.05\left(\mathrm{t}, 2 \mathrm{H}, \mathrm{J}=7.0 \mathrm{~Hz}, \mathrm{OCH}_{2}\right), 5.53\left(\mathrm{bs}, 2 \mathrm{H}, \mathrm{NH}_{2}\right), 7.04(\mathrm{~m}, 1 \mathrm{H}, 5-\mathrm{H}), \\
7.56(\mathrm{~m}, 2 \mathrm{H}, 4-\mathrm{H} \text { and } 3-\mathrm{H})\end{array}$ \\
\hline 25 & $\begin{array}{l}0.17\left(\mathrm{~s}, 6 \mathrm{H}, \mathrm{Si}\left(\mathrm{CH}_{3}\right)_{2}\right), 0.66\left(\mathrm{~m}, 10 \mathrm{H}, \mathrm{SiCH}_{2} \text { and } \mathrm{GeCH}_{2}\right), 1.02(\mathrm{t}, 9 \mathrm{H}, \mathrm{J}=7,0 \mathrm{~Hz} \text {, } \\
\left.\mathrm{GeCH}_{2} \mathrm{CH}_{3}\right), 2.63\left(\mathrm{~s}, 3 \mathrm{H}, \mathrm{CH}_{3} \text { in ring }\right), 4.05\left(\mathrm{~s}, 2 \mathrm{H}, \mathrm{OCH}_{2}\right), 5.60\left(\mathrm{bs}, 2 \mathrm{H}, \mathrm{NH}_{2}\right), 7.19(\mathrm{~m}, \\
1 \mathrm{H}, 5-\mathrm{H}), 7.68(\mathrm{~m}, 2 \mathrm{H}, 4-\mathrm{H} \text { and } 3-\mathrm{H})\end{array}$ \\
\hline
\end{tabular}

The experimental evaluation of cytotoxicity is presented in Table 5. A preliminary analysis of the structure-activity relationship for the cytotoxic action clearly indicates the strong influence of the silicon and germanium containing alkyl substituent on toxic effects in vitro. In the series of O-silyl(germyl)alkyl pyridine aldoximes and ketoximes (4-12) the $\mathrm{TD}_{50}$ values change from $1.85 \mu \mathrm{g} / \mathrm{mL}$ (silacyclopentyl derivative 6 on human fibrosarcoma HT - 1080 cell line) to non cytotoxic compounds ( $>100 \mu \mathrm{g} / \mathrm{mL}$ for 4,7 , and 8). Oxime ethers containing two elements are essentially inactive. Silacyclopentyl derivative 6 exhibits the higher cytotoxicity on $\mathrm{HT}-1080$ as compared to triethylsilylpropyl substituted pyridine aldoxime $\mathbf{5}$. For pyridine ketoxime ethers (8-10) the activity increases in order of alkyl substituents: $\mathrm{Et}_{3} \mathrm{GeCH}_{2} \mathrm{CH}_{2} \mathrm{SiMe}_{2} \mathrm{CH}_{2}$ $<\mathrm{Et}_{3} \mathrm{SiCH}_{2} \mathrm{CH}_{2} \mathrm{CH}_{2}<\left(\mathrm{CH}_{2}\right)_{4} \mathrm{SiCH}_{2} \mathrm{CH}_{2} \mathrm{CH}_{2}$. Cytotoxicity of ketoxime O-ethers is considerably lower in comparison with aldoxime O-ethers.

In the cases of O-ethers of pyridine amidoximes the highest cytotoxicity was exhibited by unsubstituted pyridine derivatives (17-20). The presence of methyl group in the pyridine ring considerably decreased the activity of amidoxime O-ethers. 
Table 5

In vitro cell cytotoxicity and the ability of intracellular NO-generation by pyridine oxime O-ethers.

\begin{tabular}{ccccc}
\hline$N^{\circ}$ & \multicolumn{2}{c}{$\mathrm{HT}$} & -1080 & \multicolumn{2}{c}{$\mathrm{MG}$} & $-22 \mathrm{~A}$ \\
\cline { 2 - 5 } $\mathbf{4}$ & $\mathrm{TD}_{50}{ }^{\mathrm{a}}$ & $\mathrm{NO}, \% \mathrm{CV}^{\mathrm{b}}$ & $\mathrm{TD}_{50}{ }^{\mathrm{a}}$ & $\mathrm{NO}, \mathrm{CV}^{\mathrm{b}}$ \\
\hline $\mathbf{5}$ & $>100$ & 3 & $>100$ & 6 \\
$\mathbf{6}$ & 3.5 & 350 & 5 & 350 \\
$\mathbf{7}$ & 1.85 & 400 & 6 & 500 \\
$\mathbf{8}$ & $>100$ & 3 & $>100$ & 4 \\
$\mathbf{9}$ & $>100$ & 8 & $>100$ & 4 \\
10 & 62 & 350 & 29 & 300 \\
$\mathbf{1 1}$ & 28 & 175 & 27.5 & 300 \\
$\mathbf{1 2}$ & 3.65 & 350 & 3.5 & 350 \\
$\mathbf{1 7}$ & 3.15 & 300 & 5.5 & 200 \\
$\mathbf{1 8}$ & 5.4 & 300 & 4.3 & 300 \\
19 & 4.85 & 250 & 24.5 & 200 \\
$\mathbf{2 0}$ & 2.5 & 550 & 4.5 & 330 \\
$\mathbf{2 1}$ & 6 & 300 & 4.5 & 300 \\
$\mathbf{2 2}$ & 21.5 & 300 & 37 & 350 \\
$\mathbf{2 3}$ & $>100$ & 9 & $>100$ & 9 \\
$\mathbf{2 5}$ & 6.35 & 250 & 22 & 5 \\
\hline
\end{tabular}

${ }^{\text {a }}$ Concentration $(\mu \mathrm{g} / \mathrm{mL})$ providing $50 \%$ cell killing effect $[(\mathrm{CV}+\mathrm{MTT}) / 2)$

${ }^{\mathrm{b}}$ NO Concentration (\%) (CV: coloration)

The NO level was determined accordingly /22/, NO release was defined using the Greyss reagent (by $\mathrm{NO}_{2}$ concentration in the cultural medium). The yield of nitrite was expressed as $\mathrm{NO}_{2} \mathrm{nmol} / 200 \mu \mathrm{L}$ of cultural medium in testing plates for $100 \%$ alive cells after CV coloration assay (pyridine derivatives concentration $100 \mu \mathrm{g} / \mathrm{mL}$ ). It was shown (Table 5) that compounds 6,9 , and 19 readily increase NO concentration in the cultural medium $\left(\mathrm{TG}_{100}=500 \%\right)$ in $\mathrm{HT}-1080$ and $\mathrm{MG}-22 \mathrm{~A}$ cell lines. 


\section{REFERENCES}

1. Scherico Ltd. Brit. Pat. 1070964 (1967); Chem. Abstr., 68,104994x (1968).

2. F.J. Villant, US Pat. 3290320 (1966); Chem. Abstr., 66, 46340b (1967).

3. C.Y. Hong, Y.K. Kim, S.H. Kim, J.H. Chang, H. Chou, D.H. Nam, A.R. Kim, J.H. Lee and K.S. Park, US Pat. 5776944 (1998); Chem. Abstr., 129, 122580s (1998).

4. T. Nishimura, C. Yamazaki and T. Ishiura, Bull. Chem. Soc. Jap., 40, 2434 (1967).

5. I. Bulete, Rom. Pat. 51935 (1970); Chem. Abstr., 73, 120678f (1970).

6. F. Rohl, V. Harries, E. Ammermann, G. Lorenz, S. Strathmann, A. Ptock, H. Sauter, W. Grammenos, T. Grote, H. Bayer, R. Kirstgen, K. Oberdorf, B. Muller and R.Muller, PCT Int. WO Pat. 9812179 (1998); Chem. Abstr., 128, 257334q (1998).

7. C. Malen, B. Danree and X.Pascaud, Ger. Pat. 2217180 (1972); Chem. Abstr., 78, 16052q (1973).

8. U. Niewoehner, U.E. Mueller, E. Perzborn, E. Bischoff and H.G. Dellweg, Eur. Pat. 471259 (1992); Chem. Abstr., 116, 214360e (1992).

9. F. Trecourt, B. Gervais, O. Mongin, C. Le Gal, F. Mongin and G. Queguiner, J. Org. Chem., 63, 2892 (1998).

10. F. Hauschild, R. Schmiedel and W.D. Wiezorek, East. Ger. Pat. 38036 (1965); Chem. Abstr., 63, 13225c (1965).

11. C. Fest, W. Brandes, G. Haenssler and P. Reinecke, Ger. Pat. 3608383 (1987); Chem. Abstr., 107, 217494b (1987).

12. W. Fuehrer, J. Stetter, H. Foerster, L. Eue and R.R. Schmidt, Ger. Pat. 3221215 (1983); Chem. Abstr., 100, 138961d (1984).

13. T. Goshima, Y. Kitagawa, S. Kaji, H. Hayakawa and A. Watanabe, Jpn. Pat. 0499767 (1992); Chem Abstr., 117, 131079q (1992).

14. R.A. Andersen, J.A.B. Barstad and K. Laake, Progr. Brain Res., 36, 189 (1972).

15. Ishikara Sangyo Kaisha, Ltd. Jpn. Pat. 8183495 (1981); Chem. Abstr., 95, 132689c (1981).

16. Farbenfabriken Bayer A.-G. Brit. Pat. 945068 (1963); Chem. Abstr., 60, 12051c (1964).

17. S. Ginsburg and I.B. Wilson, J. Am. Chem. Soc., 79, 481 (1957).

18. E. Bernasek, J. Org. Chem., 22, 1263 (1957).

19. N.S. Nametkin, K.S. Vdovin, K.S. Pushchevaya, V.I. Zavyalov, Izv. Akad. Nauk SSSR, Otd. Khim. Nauk, 1453 (1965).

20. J.W. Wilt and C.F. Dockus, J. Amer. Chem. Soc., 92, 5813 (1970).

21. K. Rubina, E. Abele, P. Arsenyan, R. Abele, M. Veveris and E. Lukevics, Metal-Based Drugs, 8, 85 (2001).

22. D.J. Fast, R.C. Lynch and R.W. Leu, J. Leuckocyt. Biol., 52, 255 (1992).

23. P.J. Freshney, Culture of Animal Cells (A Manual of Basic Technique), Wiley-Liss, New York, 1994, pp. 296-297.

24. Z. Wimmer, D. Saman, J. Smolinkova and M. Romanuk, Lieb. Ann. Chem., 1091 (1988).

25. I. Hadegorn, W.-H. Gundel, J. Hosse and Chr. Fenter, Arzneim.-Forsch. (Drug Res.)., 26, 1273 (1976). 
26. A. Steinhards and W. Mathes, US. Pat. 2924604 (1958); Chem. Abstr., 56, 736d (1961).

27. K. Rubina, Yu. Goldberg, A. Gaukhman and M. Shymanska, Synth. Commun., 19, 3129 (1989).

28. K. Rubina, Yu. Popelis, Yu. Goldberg and M. Shymanska, Chem. Heterocycl. Comp., 28, 1406 (1992).

29. E. Abele, Yu. Popelis, E. Lukevics, M. Shymanska and Yu. Goldberg, Chem. Heterocycl. Comp., 30, 14 (1994).

30. E. Abele, R. Abele, K. Rubina, J. Popelis, I. Sleikša and E. Lukevics, Synth. Commun., 28, 2621 (1998).

31. E. Ābele, R. Ābele, J. Popelis and E. Lukevics, Latv. J. Chem., N 2, 61 (1998).

32. R.J. Riddell, R.H. Clothier, M: Fd.Balls, Chem. Toxicol., 24, 469 (1986). 


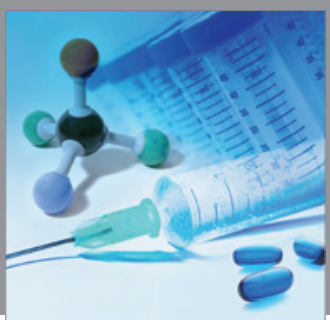

International Journal of

Medicinal Chemistry

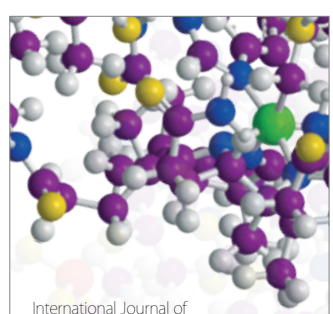

Carbohydrate Chemistry

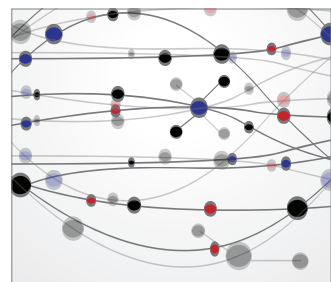

The Scientific World Journal
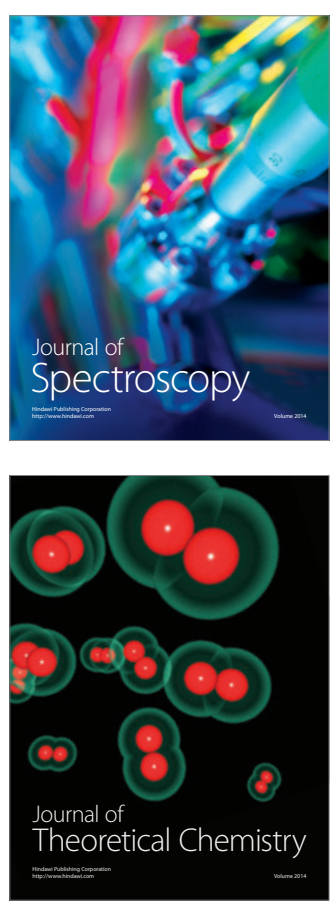
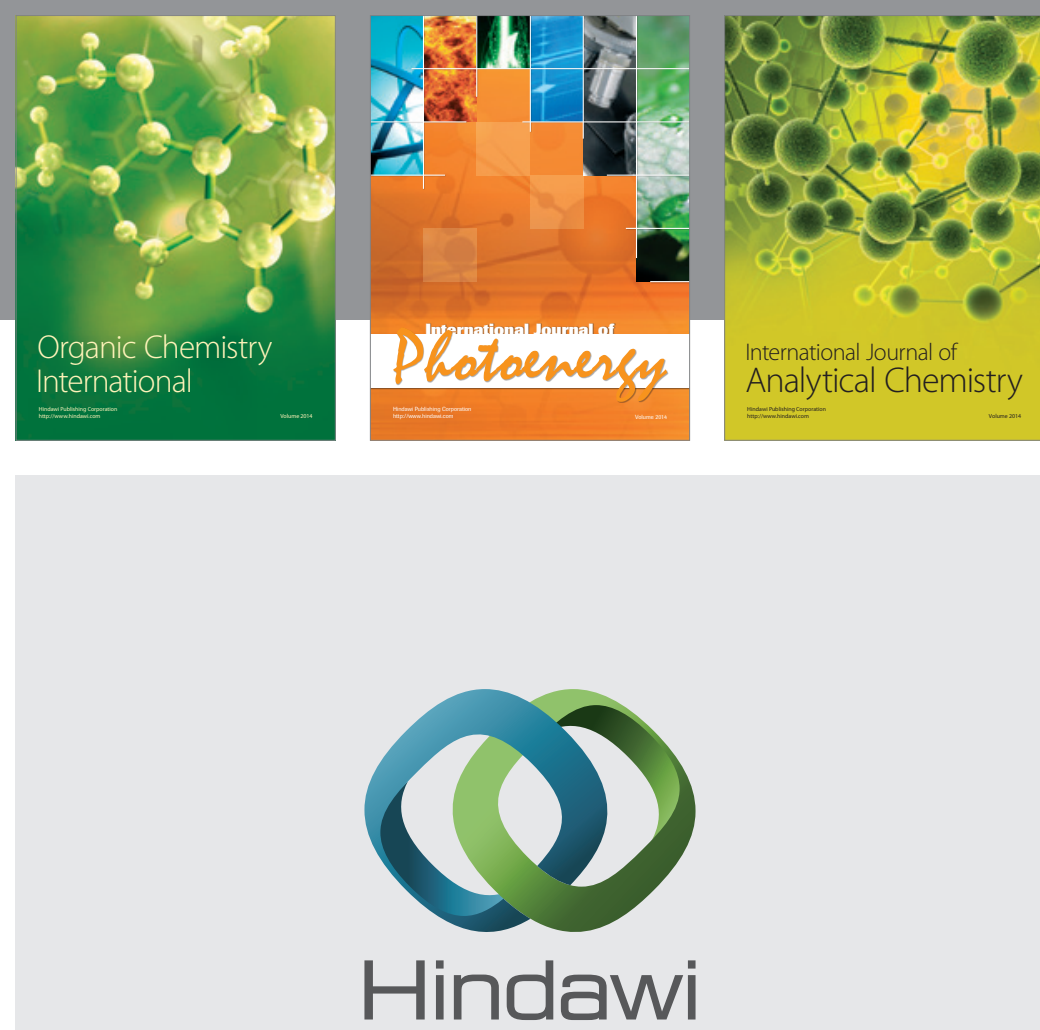

Submit your manuscripts at

http://www.hindawi.com
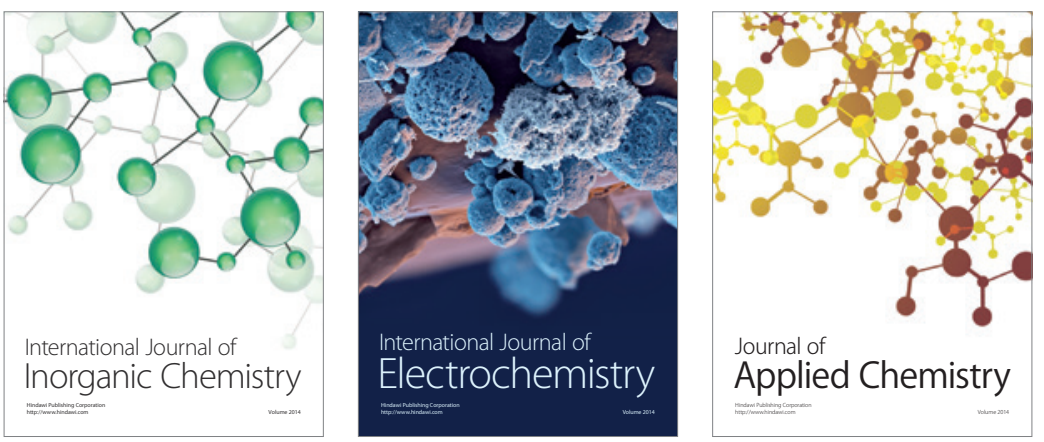

Journal of

Applied Chemistry
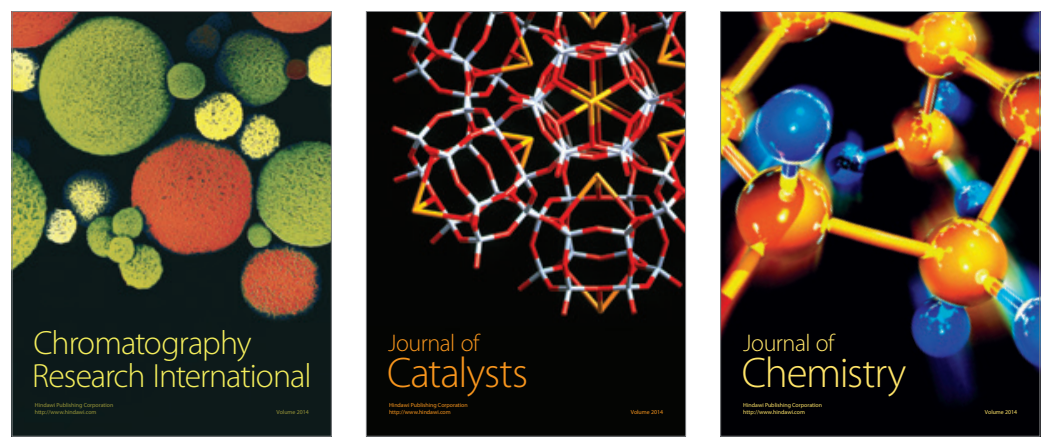
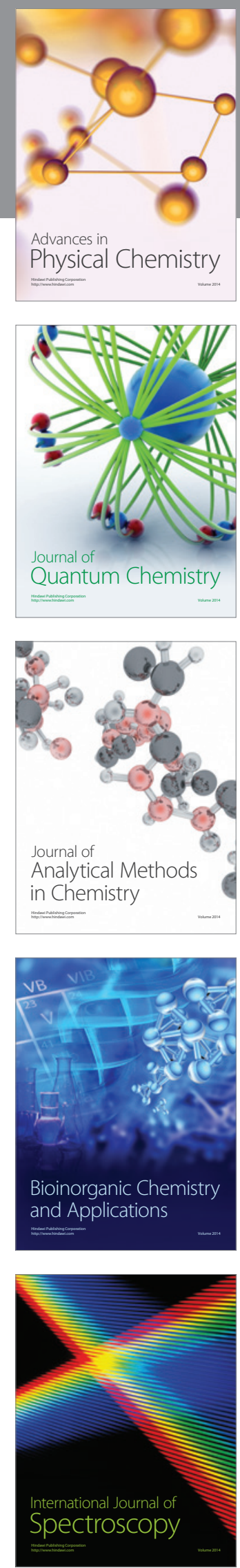